\title{
PREVALENCIA DE ENFERMEDADES BUCODENTALES EN ESCOLARES DE 6, 12 Y 15 AÑOS EN ISLAY (AREQUIPA, PERÚ)
}

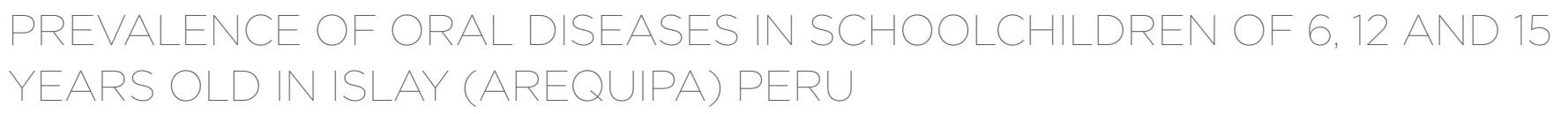

Thais Villagra-Valdivia $*$ (i)

thaisvillagra.valdivia@gmail.com

Fernando Ortiz-Culca ${ }^{2}$ (i) fernankat@gmail.com

Melvin Cisneros-del Águila ${ }^{3}$ (1) cisnerosmelvin@hotmail.com

Artículo recibido: 06/08/2019

Arbitrado por pares

Artículo aceptado: 08/10/2019

\section{* Autor corresponsal:}

Thais Villagra-Valdivia

thaisvillagra.valdivia@gmail.com

Citar como: Villagra-Valdivia T, OrtizCulca F, Cisneros-del Águila M. Prevalencia de enfermedades bucodentales en escolares de 6,12 y 15 años en Islay (Arequipa, Perú). Rev Cient Odontol (Lima). 2020; 8(1): e002. DOI: 10.21142/2523-2754-0801-2020-002

\section{RESUMEN}

Objetivo: Determinar la prevalencia de enfermedades bucodentales en niños de 6, 12 y 15 años en Islay, Arequipa. Materiales y métodos: Estudio observacional, descriptivo y transversal. La muestra estuvo conformada por 360 estudiantes de 6, 12 y 15 años, de ambos sexos, que cumplieron los criterios de inclusión. El nivel de confianza se determinó en un 95\% y el margen de error en un 5\%. Para evaluar las patologías orales, se utilizó los índices de IHO-S, IHOS-M, IPC-M, CPOD, ceod, el de la OMS y el de Dean. Los estudiantes fueron examinados visual y clínicamente. Resultados: Con referencia al IHO-S: buena higiene,72,5\% en deciduos, 63,3\% en permanentes; regular higiene, $27,5 \%$ en deciduos, $35,0 \%$ en permanentes; mala higiene, $1,7 \%$ en permanentes, existe diferencia significativa en cuanto a la edad. El CPOD fue de 6,71 cariados, 0,14 perdidos, 1,44 obturados y un promedio de 8,29; existe diferencia significativa según la edad. De acuerdo con el IPC-M, el 81,4\% padece de gingivitis; el 57,5\%, periodontitis; el 4,2\%, periodontitis severa, y hay diferencias significativas según la edad. Las maloclusiones: oclusión normal, 38,9\%; maloclusión leve, 38,6\%, y moderada o severa, 22,5\%; el 61,1\% padece de maloclusiones. Respecto de la fluorosis, el 34,7\% no presentó lesión; fluorosis cuestionable, 15\%; fluorosis muy leve, 30,3\%; fluorosis leve, 11,7\%; fluorosis moderada, 1,1\%; la prevalencia fue del 58,1\%. Conclusiones: La prevalencia de enfermedades bucodentales es elevada y el riesgo de caries dental es alto. Se observa una regular y mala higiene en la dentición permanente. El porcentaje de gingivitis es alto. Las maloclusiones están en el rango de leve a moderado. La fluorosis dental está en un rango de muy leve.

Palabras clave: índice de higiene oral, caries dental, índice CPOD, índice periodontal, maloclusión, fluorosis dental.

\begin{abstract}
Objective: To determine the prevalence of oral diseases in children 6,12 and 15 years old in Islay, Arequipa. Materials and methods: Observational, descriptive and cross-sectional study. The sample consisted of 360 students of 6,12 and 15 years of age of both sexes who met the inclusion criteria. The level of confidence was determined at $95 \%$ and the margin of error at $5 \%$. To evaluate oral pathologies, the OHI-S, OHI-M, mPI/PI, DMFT, DMFS, the WHO and the Dean index were used. The students were examined visually and clinically. Results: Regarding IHO-S: good hygiene was observed in $72.5 \%$ in deciduous and $63.3 \%$ in permanent teeth; regular hygiene was found in $27.5 \%$ in deciduous and $35.0 \%$ in permanent teeth with poor hygiene in $1.7 \%$ in permanent teeth and significant differences in age. The CPOD showed 6.71 carious, 0.14 lost, 1.44 blocked and an average of 8.29 with significant differences in relation toage. According to the IPC-M, 81.4\% had gingivitis, $57.5 \%$ periodontitis, $4.2 \%$ severe periodontitis, with significant differences for age. Malocclusions included: normal occlusion 38.9\%, mild malocclusion 38.6\% and moderate or severe $22.5 \% ; 61.1 \%$ had malocclusions. In relation to fluorosis, $34.7 \%$ had no lesion, $15 \%$ questionable fluorosis, $30.3 \%$ very mild fluorosis, $11.7 \%$ mild fluorosis, and $1.1 \%$ moderate fluorosis with a
\end{abstract}

3 Carrera de Estomatología, Universidad Científica del Sur. Lima, Perú. 
prevalence of $58.1 \%$. Conclusions: The prevalence of oral diseases and the risk of dental caries are high in children, with regular and poor hygiene being observed in permanent dentition. The percentage of gingivitis is high. The prevalence of malocclusions ranges from mild to moderate while the prevalence of dental fluorosisis is low.

Keywords: Oral Hygiene Index, dental caries, CPOD Index, Periodontal Index, malocclusion, dental fluorosis

\section{INTRODUCCIÓN}

La salud pública dirigida a la odontología basa su accionar en la prevención y control de las enfermedades bucodentales, además de la promoción de la salud bucal, a través de actividades organizadas para la comunidad. Esta rama se ocupa de la educación bucodental a la población, mediante la investigación epidemiológica y la administración de programas que ayudan a mejorar el estándar de vida de las personas. Las enfermedades bucodentales que aquejan a la población a nivel nacional pueden ocasionar diversos y serios problemas en el organismo de manera general. Entre los principales problemas bucodentales se encuentra, en primer lugar, la caries dental, que es la enfermedad más común y prevalente; en segundo lugar, la presencia de placa bacteriana, que colabora e inicia patologías que atacan a las estructuras adyacentes; en tercer lugar, la maloclusión; y, por último, la fluorosis dental ${ }^{(1-5)}$.

La información que existe sobre las enfermedades prevalentes de la cavidad bucal es variada a nivel nacional y regional, y se le da mayor importancia a la caries dental, si bien las demás (enfermedad periodontal, maloclusiones y fluorosis dental) también representan un problema de salud pública $\left.{ }^{(6-10}\right)$.

Estudios realizados en la región Arequipa, entre los años 2010 y 2018, indican que los hábitos alimenticios y la higiene bucal están relacionados con una alta prevalencia de caries dental, así como el consumo de agua tiene incidencia significativa en la presencia de fluorosis y caries dental, con predominancia de la fluorosis leve del esmalte. La alta prevalencia de gingivitis y caries dental está relacionada con los hábitos de higiene oral, los hábitos alimenticios y el nivel de conocimiento de las madres sobre estos hábitos, la cual se manifiesta en un ceod moderado y un IHOS regular (11-16).
En la misma línea de significancia de las enfermedades bucales prevalentes en la región Arequipa se encuentran las maloclusiones, para las cuales los estudios indican una predominancia de la clase I. Además, esta patología tiene una relación significativa con la caries dental $\left({ }^{17}\right)$.

El objetivo del estudio fue determinar la prevalencia de enfermedades bucodentales en niños de 6, 12 y 15 años en Islay, Arequipa.

\section{MATERIALES Y MÉTODOS}

El estudio fue de tipo observacional, descriptivo y transversal. La población estuvo conformada por 2459 niños en etapa escolar, de 6,12 y 15 años, provenientes de 66 instituciones educativas estatales y particulares de Islay (Arequipa). Este número correspondió a 849 niños de 6 años, 900 niños de 12 años y 710 niños de 15 años. Se realizaron dos tipos de determinación de muestra: el primero en función al número de colegios y el segundo para determinar la cantidad de niños. Se obtuvo un total de 9 colegios y 355 niños, que se redondeó a 360 para ambos sexos. El nivel de confianza para el estudio se determinó en un $95 \%$ y el margen de error en un $5 \%(0,05)$, de acuerdo con datos de una investigación previa $\left({ }^{16}\right)$.

Las instituciones se eligieron con relación a la cantidad de niños matriculados; los niños a evaluar por colegio se determinaron proporcionalmente por cada institución, se dividió el total de la muestra por edad entre el tamaño muestral de colegios y se obtuvo 15 niños por cada institución por cada edad. La elección de los participantes se efectuó con un muestreo aleatorio simple con la tabla de números aleatorios considerando los criterios de inclusión: niños de ambos sexos de 6, 12 y 15 años, con el consentimiento informado firmado y con 2 o más años de residencia. Se excluyó a los que presentaron enfermedad sistémica o aparatología ortopédica u ortodóncica. 

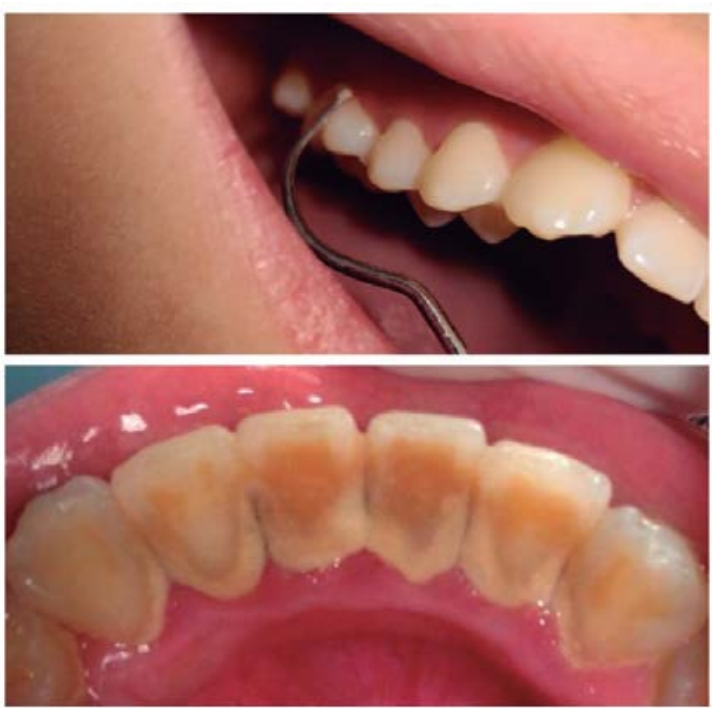

Figura I. Examen índice de higiene oral: placa bacteriana y placa calcificada

Se consideró como variables del estudio la placa bacteriana, la enfermedad periodontal, la caries dental, la maloclusión, la fluorosis, la edad y el sexo. El método utilizado fue el observacional estructurado; como técnica, el examen clínico. Para la recolección de datos se empleó una ficha preparada especialmente para este trabajo.

Como paso previo al desarrollo del estudio, la investigadora fue calibrada en un nivel gold estándar para una mejor obtención de los datos. El examen de los participantes se realizó en las primeras horas de clase para evitar el contacto con los alimentos. Para la evaluación de la placa bacteriana, se utilizó el índice de Green y Vermillion simplificado (IHO-S) y, para la dentición decidua, la modificación de Miglani, Beal, James y Behari (IHOS-M) (figura 1) $\left({ }^{17}\right)$. La evaluación de la enfermedad periodontal se realizó mediante el índice periodontal comunitario modificado (IPC-M). Se evaluó la presencia o ausencia de sangrado gingival y bolsa periodontal utilizando una sonda periodontal $\mathrm{Hu}$ Friedy-OMS, y se observó la recomendación de no realizar el sondaje para medir la profundidad de bolsa en niños menores de 15 años (figuras 2 y 3). Para la caries dental, se aplicó el índice de ceod de Gruebbel y CPOD de Klein y Palmer, mediante letras para los dientes deciduos y números para los permanentes (figura 4). Para las maloclusiones, se utilizó el índice de la OMS (figura 5) y, para la fluorosis dental, el índice de Dean (figura 6) $\left({ }^{19,20}\right)$.
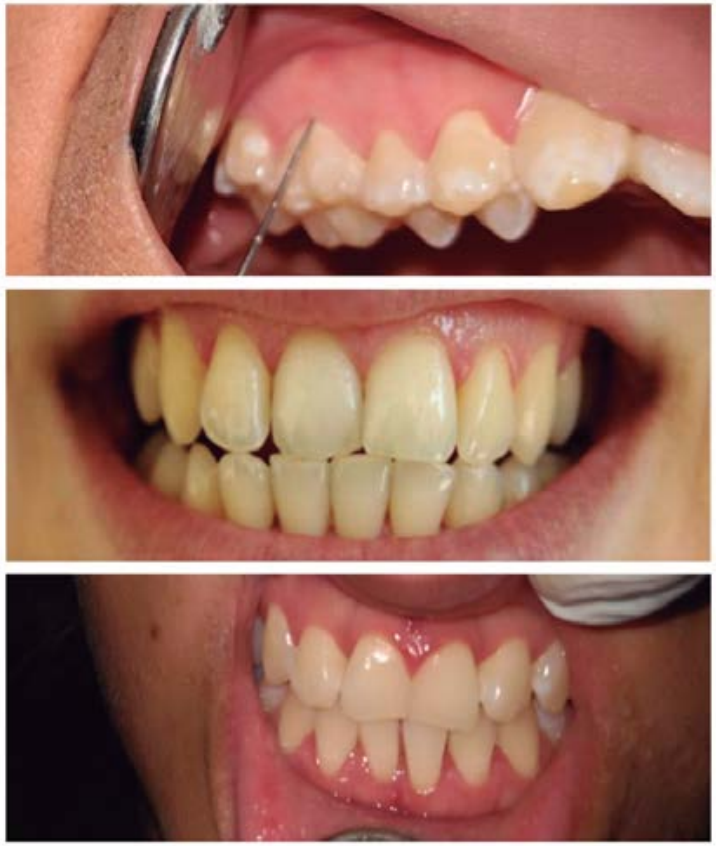

Figura 2. IPCM: persona sana, persona con enfermedad periodontal (I5 años)
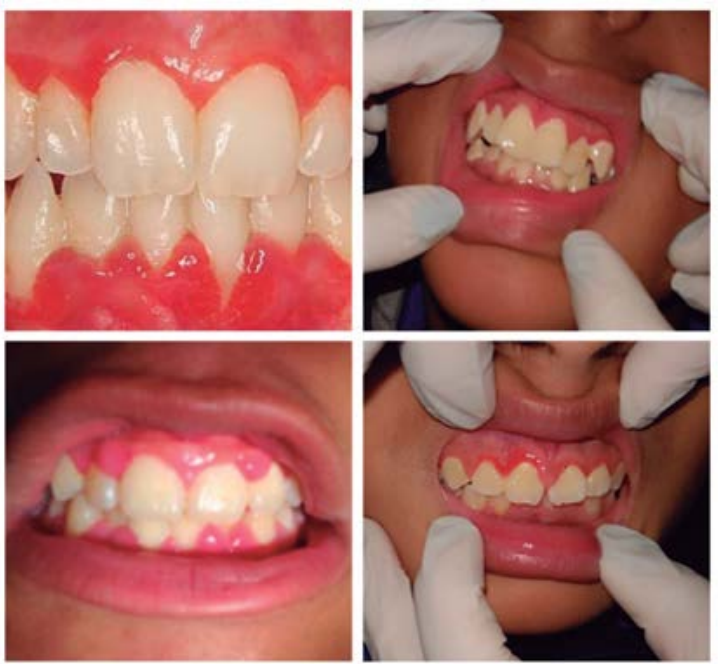

Figura 3. IPCM: gingivitis

Para el análisis univariado, se empleó estadística descriptiva, se tomaron frecuencias absolutas y porcentuales para cada una de las variables, las que fueron llevadas a una tabla de frecuencias para cada una de ellas, respectivamente. Para el análisis bivariado se determinó la asociación a partir de la prueba de chi cuadrado y $\mathrm{t}$-Student de todas las variables principales con la edad y género de la muestra en estudio. El vaciado de los datos se realizó en el programa Microsoft Excel y se analizaron 


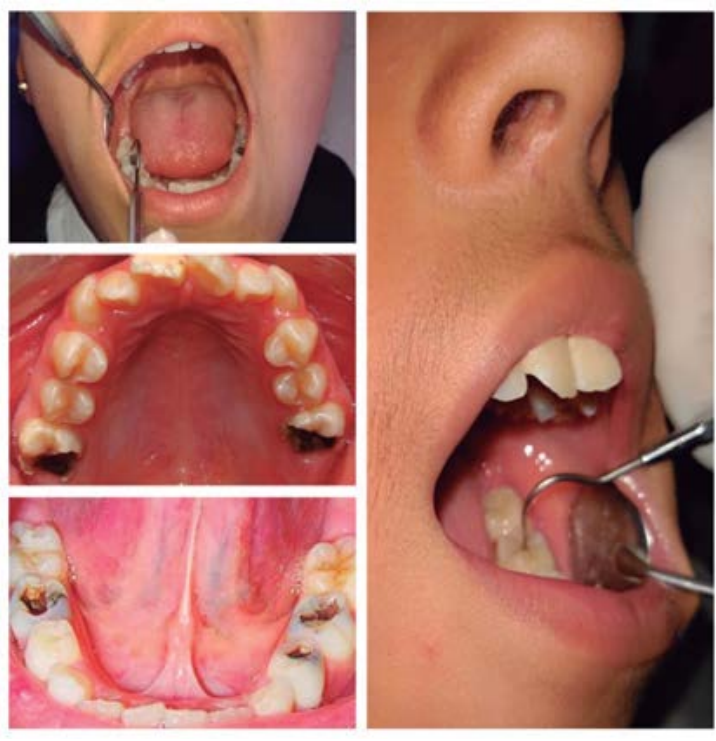

Figura 4. Índice de CPOD: persona sana, persona con caries
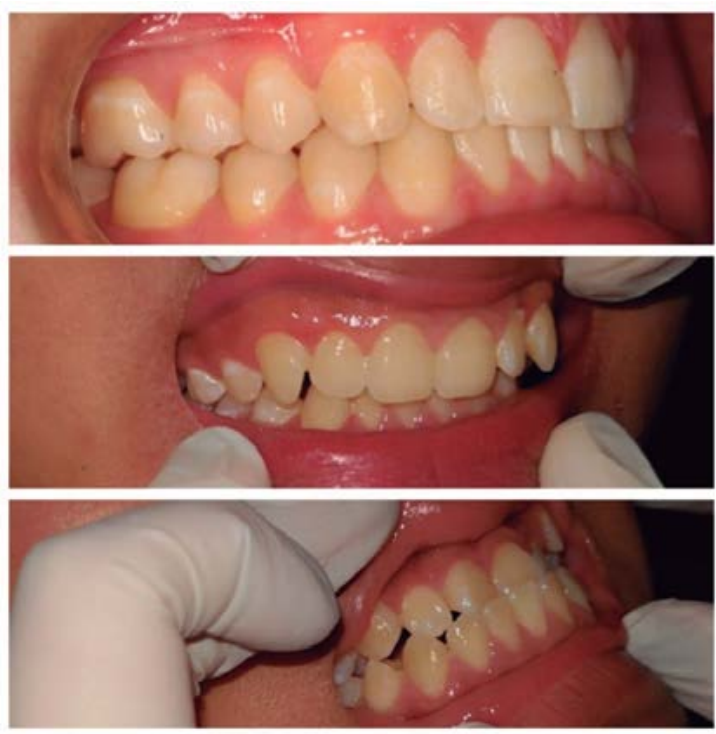

Figura 5. Índice de maloclusión de la OMS: normal, leve y moderado

los resultados mediante el paquete estadístico SPSS versión 21.

\section{RESULTADOS}

Las cifras indican que, del total de la muestra, 190 $(52,8 \%)$ correspondió al sexo masculino y 170 (47,2\%), al femenino. En referencia al índice de higiene oral simplificado, se encontró una buena higiene en $72,5 \%$

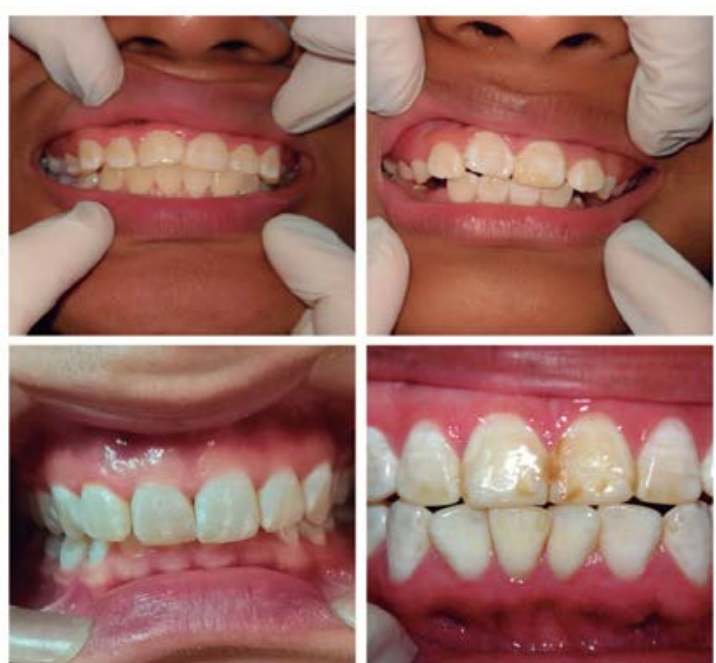

Figura 6. Índice de Dean: normal, cuestionable, muy leve, leve, moderado y grave

de dientes deciduos y 63,3\% de permanentes; regular higiene en $27,5 \%$ de deciduos y $35,0 \%$ de permanentes; la mala higiene solo se presentó en dientes permanentes en el 1,7\% de los casos. De estos datos se desprende que el promedio de regular y mala higiene fue del 36,7\%. Con relación al IHOS, existe diferencia significativa en cuanto a la edad, no así en relación con el sexo (tabla 1).

En referencia al índice CPOD, se obtuvo como promedio 6,71 para cariados, 0,14 para perdidos, 1,44 para obturados y, como promedio total, un CPOD de 8,29. Existe diferencia significativa respecto de la edad, lo que no ocurre con el sexo (tabla 2). De acuerdo con el índice periodontal comunitario, se encontró que el $81,4 \%$ de niños padece de gingivitis, el 57,5\% presenta periodontitis y el 4,2\% tiene periodontitis severa; de esto se colige que el $61,7 \%$ padece de periodontitis. Se encontró una diferencia significativa en cuanto a la edad, no así con relación al sexo (tabla 3). Con relación a las maloclusiones, se halló lo siguiente: oclusión normal, 38,9\%; maloclusión leve, 38,6\%, y moderado o severo, $22,5 \%$. Esto indica que el $61,1 \%$ padece de maloclusiones. No se encontró diferencia significativa ni para la edad ni para el sexo (tabla 4). La medición de la fluorosis dio como resultado un 34,7\% que no presentó lesión alguna, un $15 \%$ con fluorosis cuestionable, un $30,3 \%$ con fluorosis muy leve, un $11,7 \%$ con fluorosis leve y un $1,1 \%$ con fluorosis moderada. Fue excluido el $0,3 \%$ y no registrado el 6,9\%. Se encontró una prevalencia de fluorosis del 
Tabla 1. Índice de higiene oral simplificado en escolares de la provincia de Islay

\begin{tabular}{ccccc} 
Índice de higiene oral & \multicolumn{5}{c}{ Grupo dentario } \\
& \multicolumn{2}{c}{ Deciduos } & \multicolumn{2}{c}{ Permanentes } \\
& n. $^{\circ}$ & $\%$ & $\mathbf{n}^{\circ}$ & $\%$ \\
\hline Buena higiene & 87 & 72,5 & 228 & 63,3 \\
\hline Regular higiene & 33 & 27,5 & 126 & 35,0 \\
\hline Mala higiene & 0 & 0,0 & 6 & 1,7 \\
\hline Total & 120 & 100,0 & 360 & 100,0 \\
\hline
\end{tabular}

Tabla 2. Índice de CPOD en escolares de la provincia de Islay

\begin{tabular}{ccccc}
\hline \multirow{2}{*}{ Valores } & \multicolumn{5}{c}{ Caries } \\
& $\begin{array}{c}\text { Piezas } \\
\text { cariadas }\end{array}$ & $\begin{array}{c}\text { Piezas } \\
\text { perdidas }\end{array}$ & $\begin{array}{c}\text { Piezas } \\
\text { obturadas }\end{array}$ & $\begin{array}{c}\text { Índice } \\
\text { CPOD }\end{array}$ \\
\hline Media aritmética & 6,71 & 0,14 & 1,44 & 8,29 \\
\hline Desviación estándar & 3,76 & 0,50 & 2,23 & 3,82 \\
\hline Valor mínimo & 0 & 0 & 0 & 0 \\
\hline Valor máximo & 16 & 4 & 11 & 17 \\
\hline Total & & & 360 & \\
\hline
\end{tabular}

Tabla 3. Índice periodontal comunitario modificado en escolares de la provincia de Islay

\begin{tabular}{|ccc|}
\hline $\begin{array}{c}\text { TEJIDOS BLANDOS } \\
\text { ÍNDICE PERIODONTAL } \\
\text { COMUNITARIO MODIFICADO }\end{array}$ & $\mathbf{n .}^{\circ}$ & $\%$ \\
\hline Sano & 67 & 18,6 \\
\hline Con gingivitis & 293 & 81,4 \\
\hline Total & 360 & 100,0 \\
\hline BOLSA PERIODONTAL & & \\
\hline Sano & 46 & 38,3 \\
\hline Periodontitis & 69 & 57,5 \\
\hline Periodontitis severa & 5 & 4,2 \\
\hline Total & 120 & 100,0 \\
\hline
\end{tabular}

$58,1 \%$. No se encontró diferencia significativa con relación a la edad ni al sexo (tabla 5).

\section{DISCUSIÓN}

Se conoce que las enfermedades bucodentales representan un problema de salud pública; a pesar de que estamos en un nuevo siglo, las diferencias con el siglo pasado no son tan manifiestas. La caries dental, la enfermedad periodontal y las maloclusiones siguen manteniéndose en una prevalencia alta, problemas a los
Tabla 4. Índice de maloclusión de la OMS en escolares de la provincia de Islay

\begin{tabular}{ccc|}
\hline MALOCLUSIONES & $\mathbf{n .}^{\circ}$ & $\%$ \\
\hline Normal & 140 & 38,9 \\
\hline Leve & 139 & 38,6 \\
\hline Moderado/Severo & 81 & 22,5 \\
\hline Total & 360 & 100,0 \\
\hline
\end{tabular}

Tabla 5. Índice de Dean en escolares de la provincia de Islay

\begin{tabular}{|ccc|}
\hline FLUOROSIS & $\mathbf{n .}^{{ }^{\circ}}$ & $\%$ \\
\hline Normal & 125 & 34,7 \\
\hline Cuestionable & 54 & 15,0 \\
\hline Muy leve & 109 & 30,3 \\
\hline Leve & 42 & 11,7 \\
\hline Moderada & 4 & 1,1 \\
\hline Grave & 0 & 0,0 \\
\hline Excluida & 1 & 0,3 \\
\hline No registrada & 25 & 6,9 \\
\hline Total & 360 & 100,0 \\
\hline
\end{tabular}

que se ha sumado, en las últimas décadas, la fluorosis dental. Los datos obtenidos en este estudio contribuirán a mejorar el conocimiento sobre la situación de la salud bucal en esta zona del país.

Para el índice CPOD se obtuvo un promedio, el cual está considerado por la OMS como muy alto. Los datos encontrados en la Región Arequipa son, en promedio, de menor cuantía que los de este estudio; de igual manera, difieren en relación con factores para caries dental, como dieta e higiene, no así con la edad y el sexo $\left.{ }^{8,10-12,14,16}\right)$. En el presente estudio, sí hay relación significativa con la edad, pero no con el sexo. El estudio de Ponce ( $\left.{ }^{11}\right)$, en el cual los hábitos alimenticios y la higiene tienen relación con la prevalencia de caries, también difiere del presente estudio; nuestros resultados sí se asemejan a este estudio respecto de los promedios de caries 6,5 en el de Ponce y 6,71 en el nuestro). Humari $\left({ }^{21}\right)$, en la ciudad de Arequipa, encontró un CPOD de 6,11, valor muy por debajo de nuestro estudio, que es de 8,29. Esta diferencia de resultados en la misma región puede deberse al tipo de dieta y al conocimiento sobre hábitos de higiene bucal. 
El índice periodontal comunitario en nuestro estudio corresponde al $81,4 \%$ con gingivitis, similar a los resultados de Pari ( ${ }^{13}$ ), que muestran un valor del $84,4 \%$ en esta misma patología. E1 IHOS en el presente estudio es del $27,5 \%$ en deciduos y del $36,7 \%$ en permanentes de regular a mala higiene de la población total. Respecto del índice periodontal comunitario, se obtuvo un total del $81,4 \%$ con gingivitis, lo difiere con las cifras de Molina $\left({ }^{14}\right)$, que halló un $65 \%$ de niños con higiene regular, lo cual sugiere que estas cifras se relacionan con el nivel de conocimiento de las madres sobre salud oral. Consideramos que los problemas bucales son progresivos en Islay, probablemente debido a la falta de higiene (presencia de placa bacteriana) al no contar con el instrumental adecuado para la higiene bucal; además, es muy posible que la dieta no sea la correcta y no existan controles sanitarios adecuados.

En cuanto a las maloclusiones en la misma región Arequipa, los resultados de Contreras muestran un $40,3 \%$ de prevalencia, muy por debajo del presente estudio, que encontró un $61,1 \%$ de maloclusión leve a moderada. Fuera de Arequipa, en la región Ucayali, Aliaga, Mattos, Aliaga y Castillo encontraron una prevalencia de maloclusiones del 26\%. Estas diferencias pueden deberse al tipo de índice utilizado, pues los dos estudios mencionados utilizaron el de Angle y en el nuestro, el de la OMS $\left.{ }^{6,18}\right)$.

Los resultados de fluorosis en la región Arequipa, especialmente el de Manayay, cuya prevalencia corresponde al 66,3\% - ligeramente por encima del promedio del presente estudio, que es del 58,1\%-, distan de los de Guevara y Morán (9,12), que presentan un 100\% y un $95 \%$ de prevalencia en niños de similares edades. El estudio de Manayay muestra una diferencia significativa con relación al sexo, lo que difiere de nuestros resultados, en los que no se encontró ninguna diferencia en cuanto a edad ni a sexo $\left({ }^{15}\right)$.

Las limitaciones en esta investigación estuvieron relacionadas con la aceptación por parte de los padres de familia para que sus hijos participen, puesto que, tras haber solicitado el permiso a los colegios y recibidos el consentimiento informado tanto de la institución como de los padres y alumnos, la colaboración no fue total (incluidos los profesores), lo que demostró una falta de interés sobre el tema salud bucal.

Finalmente, la prevalencia de enfermedades bucodentales en niños de la provincia de Islay se mantiene en situación parecida al rango nacional rural, con la caries dental como la de mayor importancia. El riesgo para esta enfermedad en la población escolar, en promedio, es alto. La presencia de placa bacteriana en el total de la población es baja; los resultados de buena higiene son para ambos grupos dentarios, es decir, decidua y permanente. La regular y mala higiene se observa en la dentición permanente, pero en menor porcentaje. El porcentaje de gingivitis es alto en el total de la población y la presencia de enfermedad periodontal es de regular magnitud. Las maloclusiones están en el rango de leve a moderado en el total de la población. La fluorosis dental se presenta en la población en un rango muy leve.

\section{CONCLUSIONES}

La prevalencia de enfermedades bucodentales es elevada y el riesgo de caries dental es alto. Se observa una regular y mala higiene en la dentición permanente. El porcentaje de gingivitis es alto. Las maloclusiones están en el rango de leve a moderado. La fluorosis dental está en un rango de muy leve.

Contribución de autoría: Thais Villagra-Valdivia, Fernando Ortiz-Culca y Melvin Cisneros-del Águila han participado en la concepción, la recolección de información, el análisis y la interpretación de datos, su redacción y la aprobación de la versión final del artículo.

Fuente de financiamiento: Autofinanciado.

Potenciales conflictos de interés: Los autores declaran no tener conflictos de interés de ningún tipo. 


\section{REFERENCIAS BIBLIOGRÁFICAS}

1. Piédrola G. Medicina preventiva y salud pública. $12 .^{a}$ ed. Madrid: Elsevier Masson; 2015.

2. Harris N, García-Godoy F. Odontología preventiva primaria. 2. ${ }^{\text {a }}$ ed. Ciudad de México: Manual Moderno; 2015.

3. Guzmán E. Historia de la odontología. Rev Mex Odon Clín. 2006; 1 (1): 12-8.

4. Shafer W, Hine M, Levy B. Tratado de patología bucal. 4. ${ }^{\mathrm{a}}$ ed. Ciudad de México: Interamericana; 2001.

5. Cawson RA. Fundamentos de medicina y patología oral. 4. ${ }^{\mathrm{a}}$ ed. Buenos Aires: Editorial Médica Panamericana; 2006.

6. Aliaga A, Mattos M, Aliaga R, Del Castillo C. Maloclusiones en niños y adolescentes de caseríos y comunidades nativas de la amazonia de Ucayali. Rev. Peru Med Exp Salud Pública 2011; 28 (1): 87-91. doi: 10.1590/s1726-46342011000100014

7. Ortiz F. Perfil epidemiológico de salud bucal en niños atendidos en seguro social del Perú. Odontol Pediatr. 2014; 13 (2): 94-103.

8. Mayta Y. Perfil epidemiológico en salud oral en escolares de 6 a 8 años de edad de la I. E. Santo Tomas de Aquino. [Tesis de grado]. Arequipa: Universidad Católica de Santa María, Escuela Profesional de Estomatología; 2013.

9. Guevara L. Factores de riesgo de fluorosis dental en escolares de la I. E. P. N. ${ }^{\circ} 40088$, Reyno de Bélgica del distrito de Uchumayo. [Tesis de grado]. Arequipa: Universidad Católica de Santa María, Escuela Profesional de Estomatología; 2014.

10. Concha K. Índice de higiene oral simplificado en niños de 6 a 12 años de edad de la I.E. San Juan Bautista del distrito de Characato [Tesis de grado]. Arequipa: Universidad Alas Peruanas, Escuela Profesional de Estomatología; 2016.

11. Ponce C. Prevalencia de caries dental y su relación con los hábitos alimenticios y de higiene bucal en infantes de 6 a 36 meses en el programa de CRED, distritos de Hunter y Socabaya. [Tesis de grado]. Arequipa: Universidad Católica de Santa María, Escuela Profesional de Estomatología; 2010.

12. Morán A. Efecto de concentración de flúor proveniente del agua de consumo humano en la incidencia de fluorosis dental y caries dental en estudiantes de la I. E. N. ${ }^{\circ} 40672$ del asentamiento humano Villa del Triunfo del distrito de Sachaca. [Tesis de grado]. Arequipa: Universidad Católica de Santa María, Escuela Profesional de Estomatología; 2015.
13. Pari P. Prevalencia de gingivitis en niños de 6 a 12 años de edad de la I.E. San Juan Bautista [Tesis de grado]. Arequipa: Universidad Alas Peruanas, Escuela Profesional de Estomatología; 2016.

14. Molina M. Nivel de conocimiento de las madres sobre higiene bucal y hábitos alimenticios en relación con el índice de caries dental e higiene bucal de sus hijos (6-12 años) de la I. E. N. ${ }^{\circ}$ 40256 Carlos Manchego Rendón. [Tesis de grado]. Arequipa: Universidad Católica de Santa María, Escuela Profesional de Estomatología; 2016.

15. Manayay L, Castillo T. Prevalencia y grado de fluorosis en escolares de 12 años de dos colegios de Chiclayo. Revista Científica Salud y Vida Sipanense 2018; 5 (1): 27-36.

16. Nayhua L, Paredes G, Roldán L, Villavicencio P, Portocarrero J, Maguiña E. Prevalencia nacional de caries dental, fluorosis de esmalte y urgencia de tratamiento en escolares de 6 a 8,10, 12 y 15 años. Serie de Informes Técnicos de Investigación Epidemiológica. Lima: Oficina General de Epidemiologia y dirección General de Salud de las personas, Ministerio de Salud del Perú; 2005.

17. Alberto R, Flores P. Perfil epidemiológico de salud oral e indicadores de riesgo en escolares adolescentes de la localidad de Cartavio. Rev Estomatol Hered. 2010; 20 (3): 127-36.

18. Contreras L. Relación de la prevalencia de caries dental y maloclusión en niños de 6 a 12 años de la I. E. N. 40179 Teodoro Núñez Ureta. [Tesis de grado]. Arequipa: Universidad Alas Peruanas, Escuela Profesional de Estomatología; 2017.

19. Manrique JE, Manrique JA, Chávez B, Manrique C. Entrenamiento y calibración en índices epidemiológicos en salud oral. Guía Práctica. Lima; 2017.

20. Isper AJ, Saliba CA, Pantaleão MR, Elaine P. Prevalencia de maloclusión en la dentición primaria en el municipio de Cáceres, Brasil. Rev Cubana Estomatol. 2007; 44 (1).

21. Humari C. Relación de los factores asociados con la prevalencia de caries en niños de 6 a 12 años de edad que asistieron a la clínica estomatológica de la Universidad Alas Peruanas. [Tesis de grado]. Arequipa: Universidad Alas Peruanas, Escuela Profesional de Estomatología; 2016. 